Ger J Exerc Sport Res 2021 · 51:416-429 https://doi.org/10.1007/s12662-021-00770-2 Received: 7 May 2021

Accepted: 24 September 2021

Published online: 27 October 2021

(c) The Author(s) 2021

\author{
Natalie Hezel' · Carlotta Körbi' • Melissa Wolf' • Michael Adams' \\ Carl-Philipp Jansen ${ }^{2} \cdot$ Sarah Labudek' $\cdot$ Nacera Wolf-Belala ${ }^{3}$. \\ Franziska Kramer-Gmeiner ${ }^{1} \cdot$ Corinna Nerz ${ }^{2}$. Michael Schwenk ${ }^{1,4}$ \\ 'Network Aging Research (NAR), Heidelberg University, Heidelberg, Germany \\ ${ }^{2}$ Department of Clinical Gerontology and Geriatric Rehabilitation, Robert Bosch Hospital, Stuttgart, \\ Germany \\ ${ }^{3}$ Geriatric Center Ulm University, AGAPLESION Bethesda Clinic, Ulm, Germany \\ ${ }^{4}$ Human Performance Research Centre, University of Konstanz, Konstanz, Germany
}

\title{
The Lifestyle-integrated Functional Exercise (LiFE) program and its modifications: a narrative review
}

is not appealing (Costello, Kafchinski, Vrazel, \& Sullivan, 2011) and everyday activities such as gardening seem to be more suitable for being physically active in this target group (Burton, Lewin, Clemson, \& Boldy, 2013).

A particularly promising approach to counteract these barriers is the integration of specific balance and strength training into daily life activities so that no extra time needs to be reserved for training. Daily life routines can be turned into opportunities to exercise, with the long-term goal to establish more active habits. A systematic review illustrated the effectiveness of lifestyle-integrated exercises regarding improvement of motor performance and physical activity (PA) as well as fall-related outcomes in older adults (Weber et al., 2018).

In 2012, an Australian research group published an, at that time, unique and novel concept in the British Medical Journal (Clemson et al., 2012). The Lifestyleintegrated Functional Exercise (LiFE) program was originally developed for fall prevention and activity promotion in older community-dwellers ( $\geq 70$ years) by integrating functional exercise into daily routines without spending extra time (Clemson et al., 2010). Exercises for balance, strength, and PA were delivered through a one-on-one format. The LiFE program received worldwide attention and has demonstrated a reduction in the rate of falls, improvements in balance and ankle strength, an increase in selfreported PA as well as better long-term adherence compared to structured programs (Clemson et al., 2012). That is why this integrated, personal approach of LiFE has large potential to serve as an alternative to structured exercises. But, despite LiFE's numerous benefits and proven effectiveness, the original oneon-one format can only be installed with a high investment of resources which impedes large-scale implementability. Furthermore, LiFE was tested in a group of older adults without severe medical conditions. Modified programs to address the heterogeneity of needs and capabilities in the older population were therefore needed. Moreover, the LiFE concept may also be effective for preventing functional decline in young seniors, but this requires an adaption of the original LiFE program including the behavioral change concept and the development of more challenging LiFE activities.

In the past decade, several adaptions of the LiFE program have been made for tailoring the program to specific target groups ranging from young seniors to populations with cognitive or visual disabilities. Adaptions have also been made for delivering LiFE via information and 
communication technology (ICT) and administering the program in a more cost-effective group format. In addition, there is a variation of LiFE as an integrated part in a multicomponent intervention program.

The purpose of this narrative review is to provide an overview of the number of LiFE modifications and their specifications, to show their evidence regarding feasibility and effectiveness, and to discuss their challenges and potentials.

\section{The original LiFE}

In 2010 , the original, individually delivered LiFE program was developed by Clemson et al. and is the first homebased, lifestyle-integrated balance and strength exercise program for community-dwelling older adults aged 70 years and older (Clemson et al., 2010).

The LiFE activity framework involves a balance module and a strength module which are operationalized into three balance and four strength principles (Clemson, Munro, \& Fiatarone Singh, 2014a). These principles can be applied to individualized activities. The level of task difficulty depends on the individual's ability and is adapted according to the training progress. The three balance principles are reducing your base of support, shifting weight and moving to the limits of stability, and stepping over objects. One example is reducing your base of support when waiting at a bus stop by standing on one leg. The four strength principles include the following: increase the number of times using muscles; move slowly-this can make the muscles work harder; use fewer muscles to move the weight; and increase the amount of weight you have to lift or move. Performing a slow squat when getting something out of the bottom drawer is one example for the principle move slowly-this can make the muscles work harder. LiFE's initial behavior change framework (Clemson \& Munro, 2015) is based on the principles of habit formation (Lally \& Gardner, 2013). By linking the LiFE activities to daily routines, they ought to become habitual and thereby maintained in the long term.
A further goal of LiFE is the increase of PA by integrating activities for walking more and reducing sedentariness. There are also activities for reducing sedentariness, sitting less and breaking up sitting time. PA can be integrated into specific routines as well, for example getting up during TV commercial breaks.

To transfer the LiFE principles into effective activities and to adapt the training stimulus over time, LiFE is taught by trained personnel (e.g., physiotherapists) during five home visits, two booster home visits over a 3-month period and two follow-up phone calls (Clemson et al., 2014a) (• Table 1). For assistance, there is a LiFE manual for both the trainer (Clemson et al., 2014a) and the participant (Clemson, Munro, \& Fiatarone Singh, 2014b). The LiFE Assessment Tool (LAT) is a workbook which is used by the trainers at the beginning of each home visit. With the LAT, they assess participants' capability and adapt the individual exercises' levels of difficulty.

To implement this promising approach for other target groups and with less effort, five modifications of the program (group formats, for younger seniors, ICT-based formats, within multicomponent interventions, for populations with disabilities) have been developed in recent years. These modified approaches are presented below.

\section{Modifications of the LiFE program}

\section{Group formats of LiFE}

As of now, there are four group formats of the LiFE program (- Table 1). First, the idea of delivering LiFE in a group was raised by Gibbs et al. (2015) in a primary care context. Their group format of LiFE (Mi-LiFE) included four group sessions and two telephone debriefings, but still contained one individual home visit to ensure the fit of LiFE activities to participants' home environment. Further details on the intervention setting and results of their evaluation study (Gibbs et al., 2019) are listed in - Table 1. Recently, Fleig et al. (2016) published a pilot study on a group LiFE version (Everyday Activity Supports You-EASY)
Ger J Exerc Sport Res 2021 · 51:416-429 https://doi.org/10.1007/s12662-021-00770-2 (c) The Author(s) 2021

N. Hezel · C. Körbi · M. Wolf · M. Adams · C.-P. Jansen - S. Labudek - N. Wolf-Belala . F. Kramer-Gmeiner · C. Nerz · M. Schwenk

The Lifestyle-integrated Functional Exercise (LiFE) program and its modifications: a narrative review

\section{Abstract}

Lifestyle-integrated exercise is a promising approach to increase adherence levels of older adults compared to structured exercise programs as it saves time and effort and supports older adults in preventing falls and functional decline. The Lifestyle-integrated Functional Exercise (LiFE) program embodies this approach by integrating physical activity, balance, and strength activities into daily tasks of community-dwelling older adults aged $70+$. A randomized controlled trial shows strong effectiveness of the original, resource-intensive oneon-one format of the LiFE program in terms of reducing falls, improving motor performance, and increasing physical activity. The positive effects of the original LiFE program have yet stimulated adaptions to group-based and information and communications technology-based formats, to younger seniors, for multicomponent interventions, and to populations with disabilities which resulted in 16 known studies about LiFE modifications. Evidence for the effectiveness of specific LiFE modifications exists for four programs, while seven adaptions are in the feasibility stage and one is still in the early development phase. A decade of existing LiFE research is summarized in this narrative review that, to the best of our knowledge, does not exist until now. The aim of this article is (1) to provide an overview of the number of LiFE modifications and their specifications, (2) to describe the current evidence regarding feasibility and effectiveness, and (3) to present challenges and potential of the different LiFE modifications. All adaptions of the LiFE program embody the benefits of the lifestyle-integrated approach and enable an enhancement of the successful LiFE concept.

Keywords

Fall prevention - Lifestyle-integration - Habit formation · Adaption · Feasibility 


\section{Review}

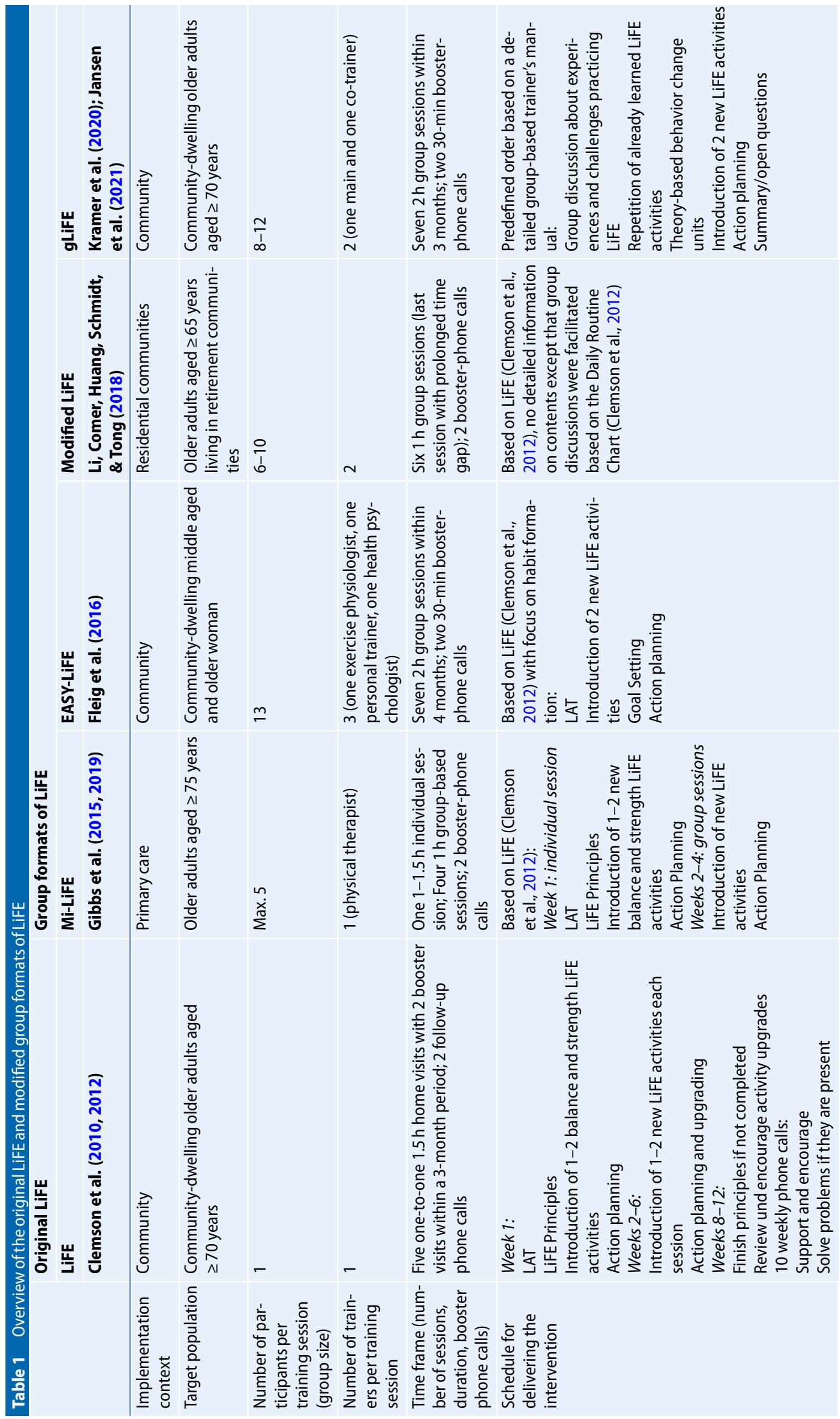




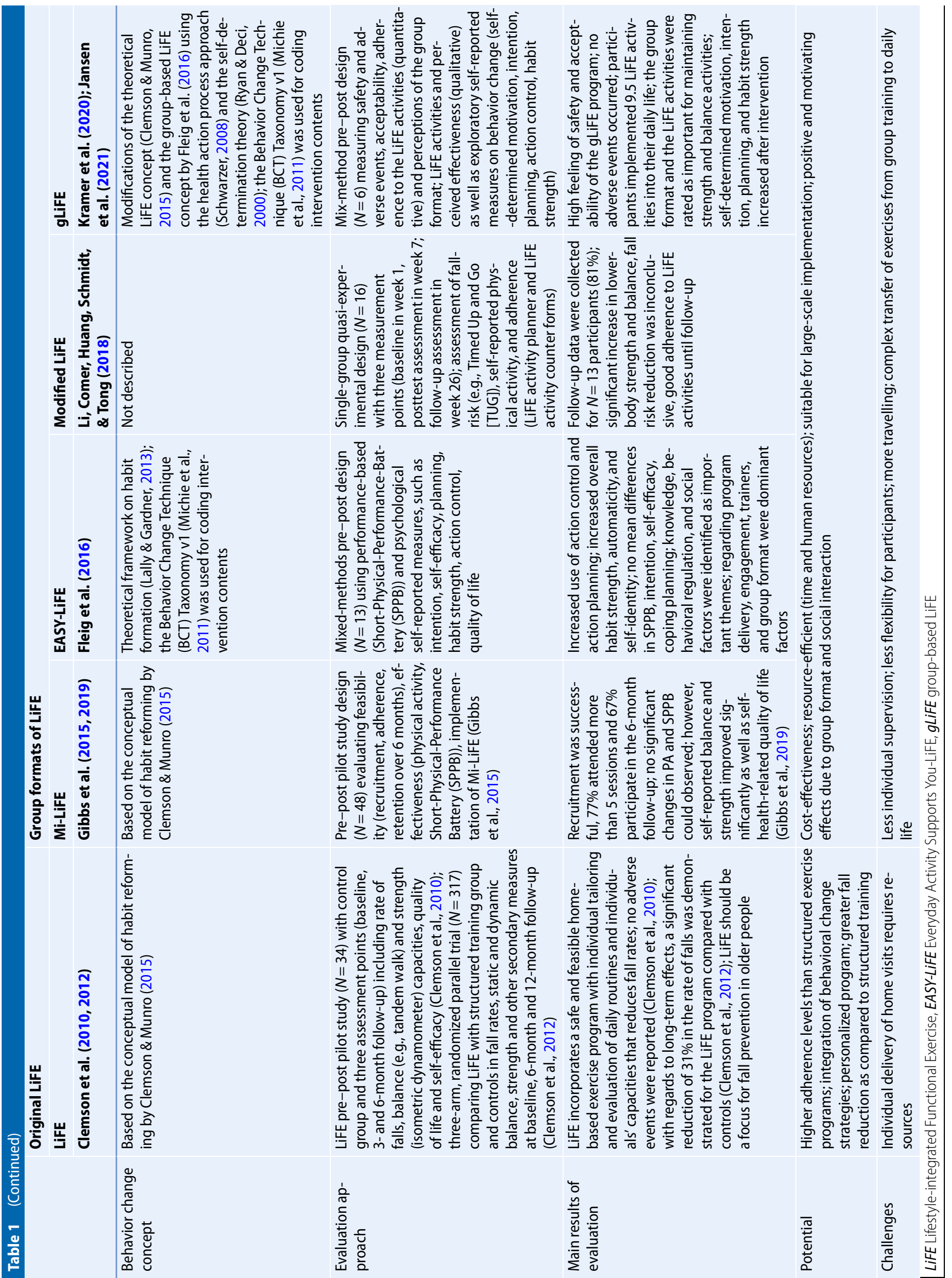


which contained an extended behavior change concept, e.g., a stronger focus on goal setting, action planning, and habit formation. In their pilot study, participants were solely female and 66 years old on average. A third pilot group LiFE version was presented by Li et al. (2018) who focused on the residential community setting. In their study, the time frame of the intervention period was extended compared to original LiFE. All these studies provided valuable input for the development of the group-based LiFE (gLiFE) format which was developed as part of the LiFE-is-LiFE project (Jansen et al., 2018), a randomized non-inferiority trial. Unlike in earlier studies (Fleig et al., 2016; Gibbs et al., 2015; Gibbs et al., 2019; Li et al., 2018), gLiFE was developed and evaluated with clear focus on large-scale implementability (Kramer et al., 2020) and compared against the original LiFE regarding effectiveness and cost-effectiveness (Jansen et al., 2021).

The main difference between LiFE and gLiFE is their delivery format ( 0 Table 1 ). gLiFE is delivered in groups of 8-12 participants and led by 2 trainers (1 main trainer and 1 co-trainer). While the single trainer in LiFE serves as a teacher and facilitator during home visits, gLiFE trainers' roles are to teach the LiFE contents and to facilitate interaction between participants. Other than the $1.5 \mathrm{~h}$ LiFE home visits, gLiFE group sessions have a duration of $2 \mathrm{~h}$. Furthermore, each gLiFE session follows a predefined order and is designed in an interactive manner including group discussions and practicing activities together with peers (Kramer et al., 2020). In comparison to LiFE where all LiFE activities are introduced in the first session, LiFE activities are introduced gradually and repeated in the subsequent session in gLiFE. This helps participants to familiarize themselves with the LiFE activities and to try them out between sessions. Furthermore, the behavior change concept was extended in gLiFE grounding on the health action process approach (Schwarzer, 2008) and self-determination theory (Ryan \& Deci, 2000). In addition, the intervention contents were coded using the behavior change techniques taxonomy (Michie et al., 2011).
In gLiFE, each participant receives the German version of the LiFE participants' manual (Clemson, Munro, Singh, Schwenk, \& Nerz, 2019) and a workbook. An activity planner and activity counter are combined in the workbook in order to simplify the planning and self-monitoring procedure (Kramer et al., 2020). In addition, specific gLiFE material was developed (e.g., posters with the LiFE principles and LiFE activities) to support group teaching.

The initial feasibility testing of gLiFE was reported jointly with the intervention concept (Kramer et al., 2020). The results showed positive group experiences and participants implemented $75 \%$ of the activities learned during gLiFE sessions, which is comparable to the adherence rate reported for LiFE (76\%). Reicherzer et al. (2021) conducted a qualitative focus group analysis that showed a high acceptance rate of gLiFE among 30 participants. gLiFE participants' attitude and motivation to participate was positively associated with learning in a group. One critical aspect of gLiFE identified in this analysis was that the implementation of activities into everyday life which was perceived as more difficult compared to LiFE.

In summary, gLiFE is the culmination of earlier pilot studies on a groupbased LiFE format with a strong focus on large-scale implementability and is the first group-based LiFE approach tested in a larger sample. The current results indicate that the gLiFE concept is a feasible and accepted alternative to the original LiFE. The publication of the main results of the LiFE-is-LiFE trial including gLiFE's potential for large-scale implementation and its cost-effectiveness are currently in preparation (Jansen et al., 2021). Several secondary analyses regarding the underlying psychological mechanisms are currently under investigation.

\section{LiFE for young seniors}

In recent years, four adapted versions of LiFE for younger seniors (EASY-LiFE, adapted LiFE [aLiFE], enhanced LiFE [eLiFE], Thai LiFE [TLiFE]) were developed to prevent early functional decline by providing an adequate level of dif- ficulty and possibilities for progression (• Table 2).

In 2016, Fleig et al. had adjusted the content of the EASY-LiFE approach to women at retirement age. Their group variation of LiFE is described in the section above and only includes women $\leq 70$ years. They highlighted this target group because the point of retirement has been shown as a good opportunity to change healthy behaviors and reduce risks for upcoming chronic diseases.

Another adaption of the original LiFE program to younger seniors, aLiFE, was developed as part of the EU-funded PreventIT project. Herein, aLiFE was created as an intervention with more challenging tasks suitable for young seniors between 60-70 years. Contrary to LiFE, aLiFE aims to prevent age-related functional decline and includes both basic and more challenging activities for individual tailoring and progression of training in young seniors. aLiFE is delivered equally to the original LiFE (Schwenk et al., 2019). Contentwise, more challenging strength and balance activities are included. By using the aLiFE Assessment Tool (aLAT), trainers can individually adjust participants' strength or balance activity levels based on a standardized procedure. In aLiFE, the new strength principle increase the range of your motion is added. Task challenge levels vary between levels 1 and 4 but are more challenging than in LiFE. Exemplary challenging strength activities in aLiFE are the one-legged squat or lunge, whereas LiFE only implements usual squats. Stepping, hopping and jumping in different ways is a new balance principle introduced in aLiFE. Through this added principle, aLiFE comprises agility activities that are based on square-stepping exercises. The seven balance activities are carried out just as in LiFE and also gLiFE. Again, more challenging balance activities such as one-leg stand with eyes closed plus cognitive task (e.g., counting backwards) are added. To reduce sitting time and interrupt sedentary phases, and to walk more, aLiFE includes structured recommendations for integrating PA into specific routines compared to original LiFE. For example, participants can park the car 


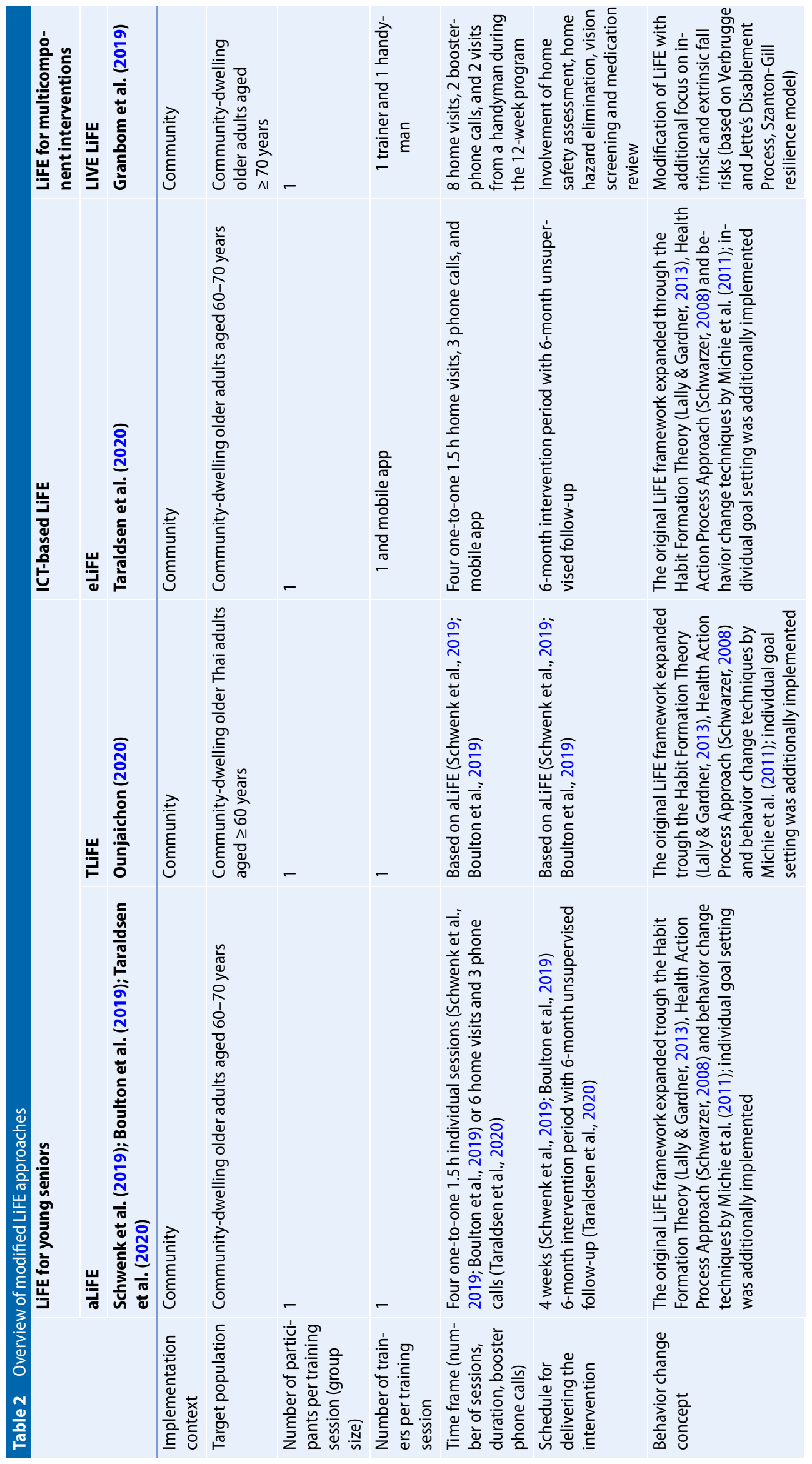




\section{Review}

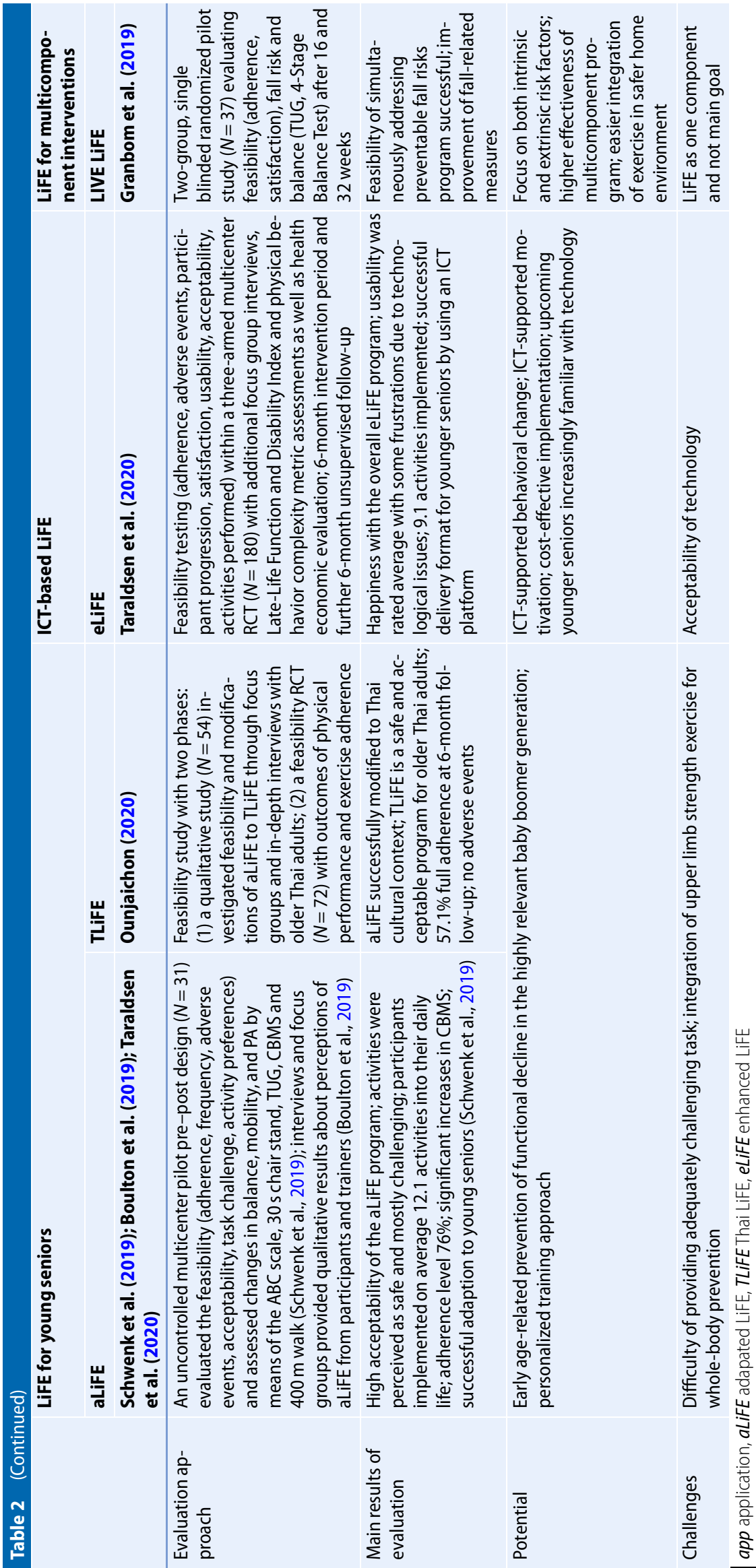


further away from the grocery store to walk more. Some adaptions were also made to the original behavioral change framework. To foster habit formation, individual activity goal setting, planning, and prompts were implemented into aLiFE.

Two further noteworthy approaches for younger seniors are modifications of the aLiFE program. Taraldsen et al. (2020) adapted aLiFE to an ICT-based delivery format (eLiFE) which will be described in detail in the following, while Ounjaichon (2020) implemented this modified version in Thailand and named it TLiFE. Adapted to the Thai lifestyle and culture, she embedded specific TLiFE activities into daily life.

Unlike EASY-LiFE which was investigated in women, the feasibility and acceptance of aLiFE was also tested in male younger seniors (Schwenk et al., 2019; Boulton et al., 2019). The aLiFE pilot feasibility trial of Schwenk et al. (2019) showed an increase in balance and mobility as measured by the Community Balance and Mobility Scale (CBMS). aLiFE participants implemented on average $12.1( \pm 1.8)$ out of 16 activities into their daily life; strength activities were most frequently implemented, followed by balance activities and PA. The frequency of practice ranged between 3.6 and 6.1 days/week depending on the activity (Schwenk et al., 2019). A study with older seniors reported 4.9 days/ week of LiFE practice (Burton et al., 2013), which means that aLiFE achieved a similar level of adherence. Within the 4-week pre-post intervention pilot study, aLiFE was additionally evaluated via interviews and focus groups with both participants and trainers. The evaluation was positive regarding participants' perception about the overall program (Boulton et al., 2019). Participants appreciated the flexible and individual approach and valued the personalized tailoring by trainers. Trainers confirmed the benefits of the delivery format to the target group. In the short 4-week timeframe, participants were not able to implement a large number of activities into their daily routines and found this burdensome. However, they were able to create new habits with some activities.
Both participants and trainers reported extensive study paperwork but changes to this had already been made reflecting suggestions after this pilot study.

The acceptability and feasibility of the TLiFE program was investigated in older adults aged $60-75$ years. The majority of the participants were fully $(57.1 \%)$ or partially $(40.0 \%)$ engaged in TLiFE after 6 months. Furthermore, results suggested that the TLiFE program was acceptable and safe for conducting a larger RCT for further evidence (Ounjaichon, 2020).

In summary, the transfer of LiFE to the population of young seniors seems to have been successful resulting that lifestyle-integrated functional exercises do not have to be limited to simple activities as in original LiFE but can also include more complex activities promoting the prevention of earlier functional decline in younger seniors.

\section{ICT-based LiFE}

To date, there is one existing ICT-based LiFE format. Enhanced by an eHealth smartphone and smartwatch-based system, the eLiFE program combines behavioral changes and ICT in a further implicit approach of integrating functional exercises into daily routines targeting younger seniors (Taraldsen et al., 2019).

The eLiFE program implements the same lifestyle-integrated exercise intervention as aLiFE but differs in the delivery format: eLiFE is enhanced by a smartphone- and smartwatch-based system in which contents are delivered through video clips, pictures, and text/ verbal instructions for each activity. eLiFE provides motivational messages and real-time feedback to the users to keep them engaged in the program. According to their individual progress, eLiFE participants receive tailored recommendations to upgrade their activities via app compared to a series of home visits and phone calls as in aLiFE. eLiFE instructors only support participants to understand and undertake the program in four home visits so that they learn how to set goals and include activities into daily life situations (• Table 2).
In a multicenter, feasibility RCT in three European countries (Norway, Germany, Netherlands) as part of the European PreventIT project, 180 younger seniors (ages 61-70 years) were randomly assigned to either eLiFE, aLiFE, or control group (Taraldsen et al., 2020). The feasibility of the digital technology delivery format of eLiFE was investigated and both programs were compared. Within the 12-month intervention duration, participants were supported by a trainer for the first 6 months and were then encouraged to continue with their individual activity program for the following 6 months (unsupervised follow-up). aLiFE and eLiFE indicated new habit formation during the first period which were sustained at follow-up. eLiFE's overall concept was generally well accepted. However, some challenges due to technical issues of the delivery format were reported. Improvement in clinical outcomes was found in all groups, but no significant differences in change between the three groups (Taraldsen et al., 2020).

In conclusion, results suggest that functional and behavioral changes can be achieved through different pathways. It was shown that the ICT-based eLiFE enriches daily routines by challenging participants' balance and strength capacity.

\section{LiFE within multicomponent interventions}

There is one existing adaptation of LiFE within a multicomponent intervention, which is called LIVE LiFE (• Table 2). Like the original LiFE, the program also targets community-dwelling adults aged 70 years and older. However, LIVE LiFE not only includes balance and strength training, which is integrated into daily routines, but also an additional individualized home safety assessment, home hazard elimination, vision contrast screening, and a medication review. Thus, this program incorporates both intrinsic and extrinsic fall risks (Granborn et al., 2019). Results from an initial pilot study showed that all fall-related measures in the intervention group improved after 16 weeks. A statistically significant difference between the intervention and con- 
trol group could be shown in tandem standing time for example which provides evidence for further investigations (Szanton et al., 2021).

\section{LiFE for older adults with disabilities}

Older adults with cognitive, visual, or mobility disabilities are at particular risk of functional decreases (van Alphen et al., 2016; Ivers, Cumming, \& Mitchell, 2003). Currently, there are four modified versions of LiFE for populations with disabilities (『 Table 3): LiFE for people with dementia (LiFE4D), LiFE for people with dementia in acute care (diaLiFE), LiFE for people using Restorative Home Care Service and LiFE for older adults with visual impairments (v-LiFE).

One of these modifications was developed by Almeida et al. (2020) who adapted LiFE for people with dementia (LiFE4D). In addition to the already included activities for lower limb strength and balance in the original LiFE, they complement components for upper limb functionality, flexibility, and cardiorespiratory endurance. Moreover, educational and psychosocial components, such as talks and flyers on dementia-related topics (e.g., fall prevention, communication, support in the community) are included. The program lasts 3 months and comprise face-to-face sessions in a familiar environment of about $1 \mathrm{~h}$ and telephone calls of no more than $15 \mathrm{~min}$. It starts with three weekly face-to-face sessions in the first month and ends with one weekly face-to-face session and one phone call biweekly in the last month and finally only two phone calls in the last week (Almeida et al., 2020). Belala et al. (2019a) took a first step to develop an early rehabilitation and transitional care intervention for people with dementia in acute care (diaLiFE). They investigated the feasibility and acceptability of the original LiFE exercise framework in cognitive impaired people in a clinical setting. Burton et al. (2013, 2014a, b) modified the LiFE program for the use in a restorative home care setting. Restorative home care services are short-term services for 6-12 weeks with the goal of helping older people regain function and regain or maintain independence, often after hospitalizations. For this purpose, the paperwork is adjusted to save time for the care managers. In addition, instead of five sessions in original LiFE, participants receive three sessions over the time. Within these three sessions, the LiFE program is only one aspect of their service. Keay et al. (2015) reported an adaptation and implementation of the LiFE program as a fall prevention strategy for adults over 50 years with visual impairments ( $\mathrm{v}$ $\mathrm{LiFE}$ ). Due to visual limitations, the program is delivered by orientation and mobility (O\&M) specialists (Dillon, Clemson, Coxon, \& Keay, 2018). Just like the original LiFE program, v-LiFE consists of three balance and four strength principles. The program is delivered through five sessions weekly, two booster visits within 3 months, two support services after the visits, and two additional sessions if needed. In support of this, v-LiFE participant's manual is created specifically for this target population in audio, large print, and electronic formats (Keay et al., 2018).

A number of studies investigated feasibility, acceptability and effectiveness of these modified LiFE versions for older adults with disabilities. In a pilot study, Almeida et al. (2021) explored feasibility and preliminary effectiveness of LiFE4D in 12 sedentary people with minor to major neurocognitive impairment. The authors reported an average adherence of $95.6 \%$ to the sessions. Potentially relevant functional progress in the LiFE group was found for cardiorespiratory endurance and balance. However, the group was too small to actually be able to make statements about effects. Numerous other measurements (e.g., upper and lower limb muscle strength) also showed no effect (Almeida et al., 2021). Belala et al. (2019a) recruited 20 inpatients from a subacute geriatric rehabilitation center with mild to moderate cognitive impairment. In this pilot study, they found a good acceptance rate for the overall activity framework in cognitive impaired people in a clinical setting. However, the activities were only feasible under supervised conditions and with support by trainers. The LAT was conducted successfully, but floor effects occurred in every activity. This reflected that the activities were too demanding for part of the subjects and must be started at a lower level. Accordingly, adjustments of LiFE activities are needed before it can be applied in a hospital population. The amount of adjustment is probably dependent on the target population of inpatients. In order to avoid floor effects and enable an autonomous and safe training, even lighter exercises should be integrated into the program, e.g., heel raises (calf raises) in standing position with both hands on a wall instead of walking on heels. To obtain further information for developing an adapted activity framework, Belala et al. (2019b) conducted an observational study to analyze activity behaviors and reasons for physical inactivity among cognitively impaired hospitalized individuals. Results revealed that subjects were inactive for more than $85 \%$ of the observed time and that sedentariness was linked to the time spent alone, to the presence of neuropsychiatric symptoms and to the daytime. While most therapeutic and nursing activities took place in the morning, there was a lack of activities in the afternoon, which could be used for the implementation of LiFE activities. LiFE was also found to be feasible for inclusion in a restorative home care setting (Burton et al., 2014a). There were no differences between groups in adherence, but significantly better outcomes for the LiFE group in $40 \%$ of the measures (Burton et al., 2013). Six months later, this effect was still evident in $25 \%$ of the outcome measures (Burton et al., 2014b). Therefore, the inclusion of LiFE instead of classical exercise programs might demonstrate a possible alternative for organizations that provide restorative home care services in order to improve functional outcomes.

$\mathrm{v}$-LiFE was evaluated regarding feasibility and acceptability in a pilot study (Keay et al., 2015). Significant positive effects were obtained in fall rates and function, making the program a promising approach to improve balance and strength in adults with visual impairment (Keay et al., 2015). Improvements in the pilot evaluation supported the plan to conduct a large-scale trial (pragmatic RCT) to evaluate the effectiveness, cost- 


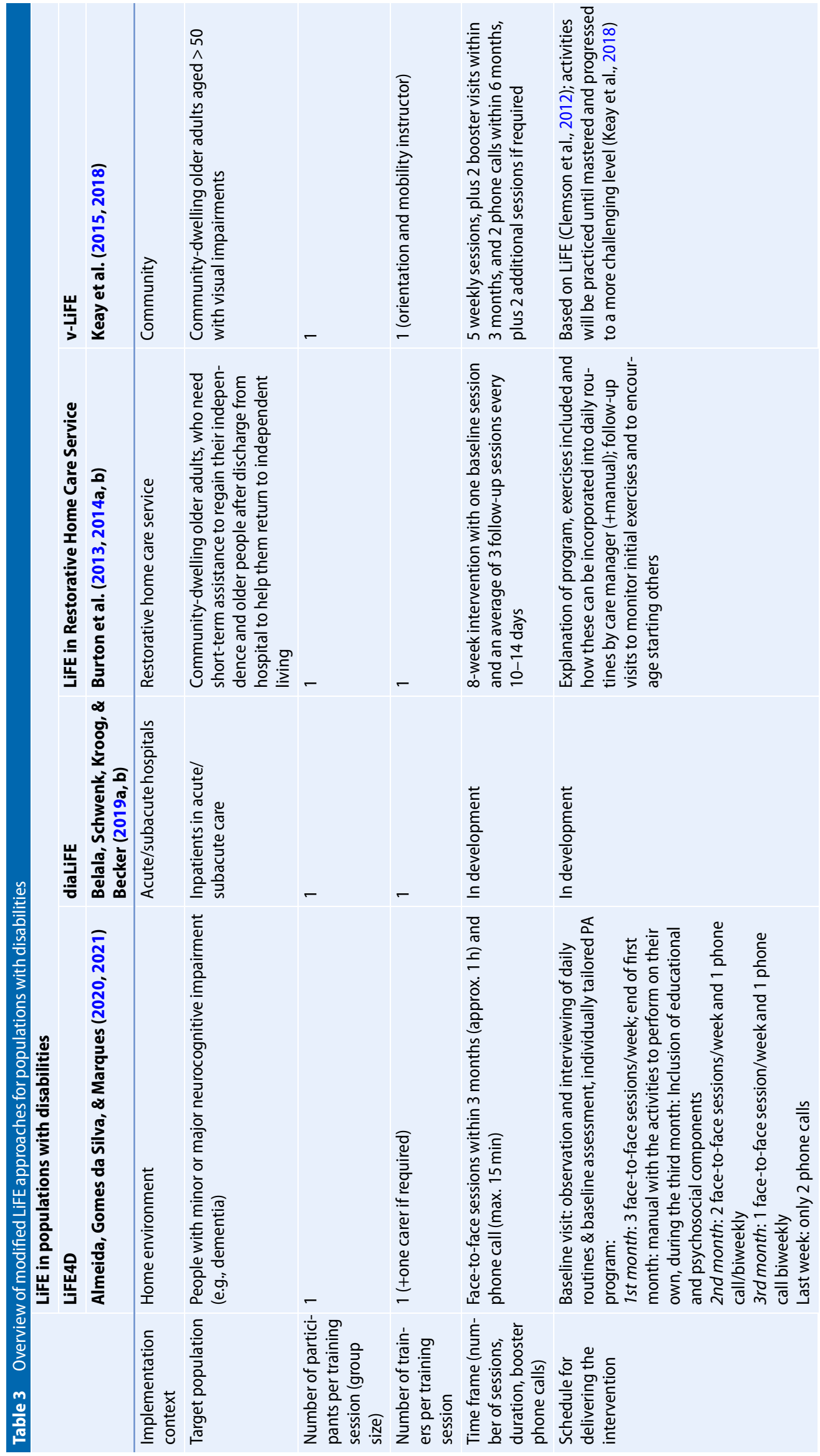




\section{Review}

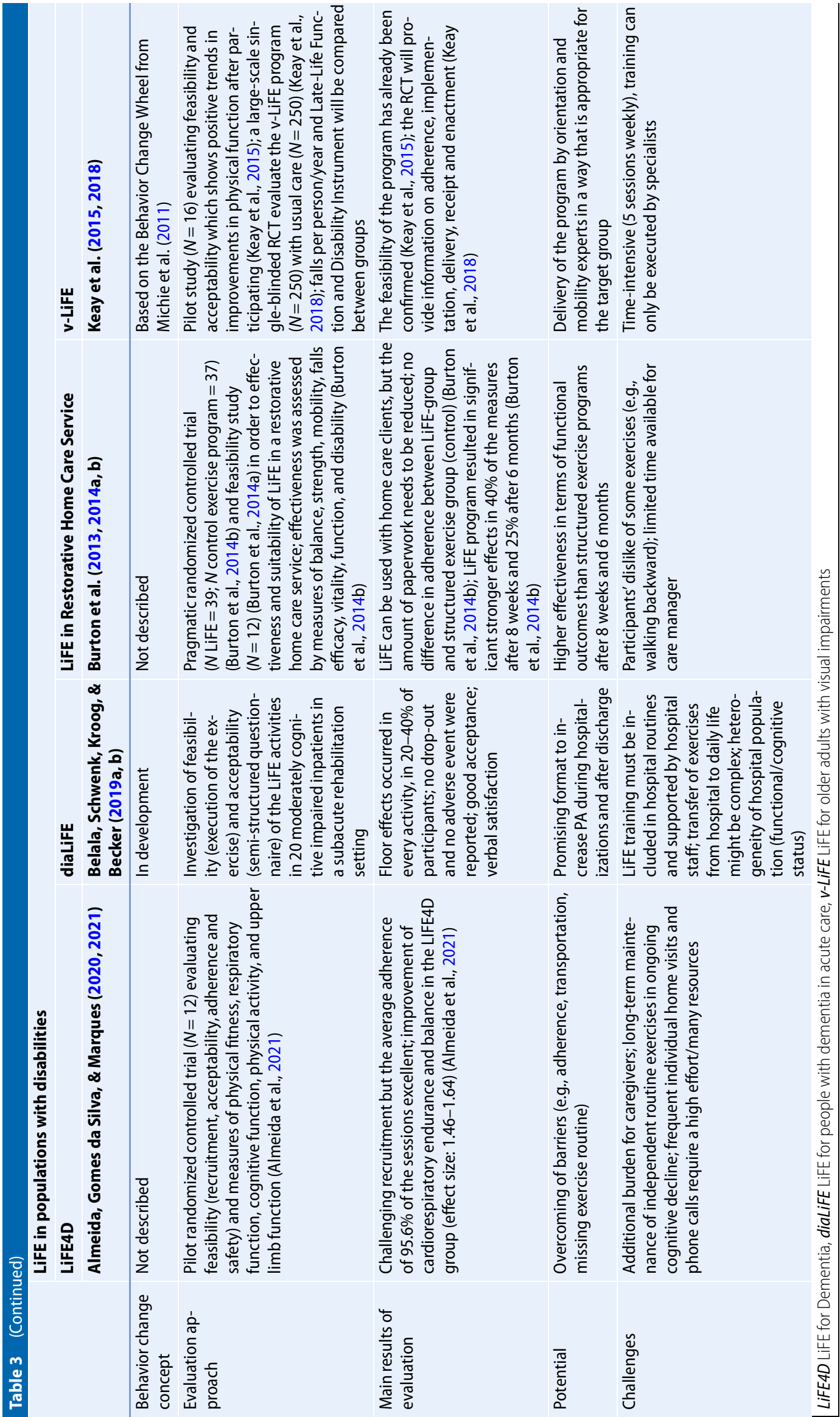


effectiveness, and implementation of this strategy (Keay et al., 2018). Both quantitative and qualitative data are processed for the process evaluation (Dillon et al., 2018). The results of the process are currently pending.

In summary, modified versions of the LiFE program have been shown partial evidence for the feasibility, acceptance, and effectiveness in different populations with disabilities and different settings. Further studies are needed to explore whether clinical relevant effects can be achieved in terms of health and quality of life and to compare them to those of structured exercise programs.

\section{Discussion}

\section{Short summary of the modifications and adaptions}

This article shows that several modifications of the LiFE program have been made and evaluated since the development of the original LiFE program by Clemson et al. in 2010. Modifications range from different delivery formats as group approaches to ICT-based programs implemented using a mobile app (application). In addition, adaptions were made to reach younger target groups as well as older adults with disabilities. The numerous modifications of the original LiFE program highlight the high need for effective falls prevention for the heterogeneous population of older adults.

\section{Current evidence regarding feasibility and effectiveness}

So far, the original LiFE program is best investigated with respect to effectiveness regarding falls rate and physical function. A RCT with a large sample $(N=317)$ showed excellent feasibility and positive effects on balance, strength, and fall rates in adults aged 70 years and older (Clemson et al., 2012). The reduction in falls of $31 \%$ exceeds the average fall reduction described in Cochrane reviews. To date, several studies suggest (e.g., Gibbs et al., 2019; Kramer et al., 2020) that the feasibility of group formats of LiFE is comparable to the original format in terms of adherence rates, safety, and acceptability.
Most recent findings of a randomized non-inferiority trial suggest that gLiFE is comparably effective regarding functional outcomes and strongly improves physical activity to a greater extent than LiFE. On the other hand, non-inferiority regarding physical activity-adjusted fall risk was inconclusive (Jansen et al., 2021).

The adaption to young seniors (aLiFE) is in the feasibility stage. Current studies show promising results with respect to feasibility for short-term (Schwenk et al., 2019; Boulton et al., 2019) and long-term interventions (Taraldsen et al., 2020). Pilot studies indicated effects on balance and mobility (Schwenk et al., 2019) and changes in PA patterns (Zhang et al., 2018), event after a short period. However, evidence is low due to uncontrolled study designs. On the same note, no significant differences between groups were found in primary outcomes in a largescale RCT (Taraldsen et al., 2020), suggesting that aLiFE needs to be refined to include more appropriate task challenges. The implementation and feasibility in the Thai context has been investigated and confirmed so far (Ounjaichon, 2020). Further studies evaluating the effectiveness of this version (TLiFE) are needed.

Participants' overall satisfaction with the ICT-based eLiFE was shown in the PreventIT project in which delivery of a 6month lifestyle-integrated intervention via ICT was as successful as via the traditional paper-based format (Taraldsen et al., 2020). However, primary outcome measures of this RCT did not differ between intervention groups and controls, again highlighting the need for program refinement to improve effectiveness.

Feasibility of a multicomponent LiFE format was demonstrated by Granbom et al. (2019). The multicomponent LIVE LiFE format improved all performance measures with small to large effect sizes, showing significant differences between the intervention and control group (Granbom et al., 2019). This pilot study provided a good basis for largescale studies.

Overall, the results of the modified LiFE formats for populations with disabilities suggest good feasibility, i.e., high adherence rates were shown for peo- ple with dementia in LiFE4D. However, the small sample size of the pilot study (Almeida et al., 2020) diminishes the power of the results and more robust methods are required in further investigations. DiaLiFE, as a clinical setting approach, is still in the development stage. To date, it is unclear whether the hospital setting is appropriate to facilitate behavior change with respect to PA. However, it might present an important part of future transitional care interventions. Burton et al. (2013) investigated the feasibility of the LiFE program in a Restorative Home Care Service. Participants disliked some of the exercises but were still able to perform them correctly (Burton et al., 2013). Nonetheless, the positive short-term as well as long-term effects are predominating and promote the aim of increased PA (Burton et al., 2014b). v-LiFE is the first fall prevention program for the visually impaired, filling a major gap in current orientation and mobility services (Keay et al., 2018). Should the results of the pragmatic RCT $(N=500)$ show v-LiFE's effectiveness and efficacy, both individuals and the health care system may benefit.

\section{Challenges and potential for future LiFE modifications}

The main potentials of LiFE are the integrated, personal approach, adjusted to the individual daily schedule although the personalized approach comes with a challenge: implementing LiFE during seven home visits requires high resources and thereby hampers large-scale implementation. Although group-based LiFE formats might be able to facilitate costefficient implementation of LiFE, participants might struggle with meeting at fixed appointments or transferring exercises from group training to daily routines at home. On the other hand, the groupbased delivery could come with benefits through presence of peers and a sense of relatedness.

The aLiFE adaptation seems to be a time-efficient program for preventing functional decline in young seniors but has difficulty with providing adequately challenging tasks. TLiFE provides the same potential for younger Thai seniors. The transfer to the ICT-based eLiFE 
format for younger seniors has realistic potential for a simplified and even costeffective delivery. Nevertheless, technical issues with the mobile app required additional home visits during the intervention period and need to be considered and revised.

The potential of the multicomponent LIVE LiFE program lies in combining adapted components. However, the cost factor is a particular challenge of the program.

LiFE for populations with disabilities are useful extensions of the program. While LiFE4D shows additional potential for improving health-related cardiorespiratory fitness and balance for people with dementia, diaLiFE is a promising format to increase PA during hospitalizations and after discharge. However, one key question of the program is whether the program results in inpatients can be successfully transferred from the hospital to the home environment. With LiFE in Restorative Home Care Services, there is an additional approach promoting the regain of independence. But time constraints of caregivers pose immense challenges to the successful and effective implementation of this format. Also, the time-intensive referral process by specially trained orientation and mobility specialists presents a unique challenge for v-LiFE. Nevertheless, this visually impaired group with high risk of falls needs to be considered in future modifications.

\section{Limitations}

Although the present article provides guidance for researchers and practitioners on content and choice of suitable LiFE formats, some limitations to the present research should be considered. This article provides a comprehensive overview but is not a systematic review. Due to the fact that no systematic search was carried out, relevant work related to LiFE could have been missed. Moreover, we could not include and explain more approaches of LiFE (e.g., Hu, Keglovits, Somerville, Snyder, \& Stark, 2020) due to the limited scope of this article.

\section{Conclusion}

This narrative review provides an overview of the original LiFE program by Clemson et al. (2012) and selected modifications of this intervention approach. The overall effectiveness of the program has been established, and results on the feasibility of adaptations to different target populations are promising, ranging from the "young old" to older adults and specific populations such as older adults with visual or cognitive impairment. Future research needs to test the effectiveness of those modifications in larger trials and based on more rigorous methodology. In addition, it can be positively emphasized that modified LiFE programs also achieve higher adherence levels than structured programs and that more cost-effective approaches are well implementable.

Finally, it should be particularly highlighted that the modifications of LiFE build on the benefits of the lifestyle-integrated approach, with most being less resource-intensive and taking older adults with special needs or capabilities into account.

\section{Corresponding address}

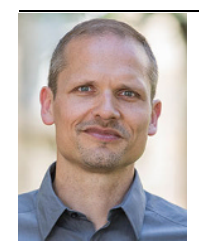

Dr. Michael Schwenk

Network Aging Research (NAR), Heidelberg University Bergheimer Straße 20, 69115 Heidelberg, Germany schwenk@nar.uniheidelberg.de

Funding. Open Access funding enabled and organized by Projekt DEAL.

\section{Declarations}

Conflict of interest. N. Hezel, C. Körbi, M. Wolf, M. Adams, C.-P. Jansen, S. Labudek, N. Wolf-Belala, F. Kramer-Gmeiner, C. Nerz and M. Schwenk declare that they have no competing interests.

For this article no studies with human participants or animals were performed by any of the authors. All studies performed were in accordance with the ethical standards indicated in each case.

Open Access. This article is licensed under a Creative Commons Attribution 4.0 International License, which permits use, sharing, adaptation, distribution and reproduction in any medium or format, as long as you give appropriate credit to the original author(s) and the source, provide a link to the Creative Commons li- cence, and indicate if changes were made. The images or other third party material in this article are included in the article's Creative Commons licence, unless indicated otherwise in a credit line to the material. If material is not included in the article's Creative Commons licence and your intended use is not permitted by statutory regulation or exceeds the permitted use, you will need to obtain permission directly from the copyright holder. To view a copy of this licence, visit http://creativecommons.org/licenses/by/4.0/.

\section{References}

Almeida, S., Gomes da Silva, M., \& Marques, A. (2020). Lifestyle integrated functional exercise for people with dementia: study protocol for a home-based randomised controlled trial. International Journal of Therapy And Rehabilitation, 27(5), 1-14.

Almeida, S., Paixão, C., da Silva, M. G., \& Marques, A. (2021). Lifestyle-integrated functional exercise for people with dementia: a pilot study. Journal of Aging and Physical Activity, 1, 1-10.

Bauman, A., Merom, D., Bull, F.C., Buchner, D. M., \& Singh, F.M.A. (2016). Updating the evidence for physical activity: summative reviews of the epidemiological evidence, prevalence, and interventions to promote "active aging". Gerontologist, 56(2), 268-280.

Belala, N., Schwenk, M., Kroog, A., \& Becker, C. (2019a). Feasibility of the lifestyle integrated functional exercise concept in cognitively impaired geriatric rehabilitation patients. Zeitschrift für Gerontologie und Geriatrie, 52(01), 61-67.

Belala, N., Maier, C., Heldmann, P., Schwenk, M., \& Becker, C. (2019b). A pilot observational study to analyze (in)activity and reasons for sedentary behavior of cognitively impaired geriatric acute inpatients. Zeitschrift für Gerontologie und Geriatrie, 52, 273-281.

Boulton, E., Weber, M., Hawley-Hague, H., Bergquist, R., Van Ancum, J., Jonkman, N. H., et al. (2019). Attitudes towards adapted lifestyle-integrated functional exercise developed for 60-70-yearolds: perceptions of participants and trainers. Gerontology, 65(6), 599-609.

Burton, E., Lewin, G., Clemson, L., \& Boldy, D. (2013). Effectiveness of a lifestyle exercise program for older people receiving a restorative home care service: a pragmatic randomized controlled trial. Clinical Interventions in Aging, 8, 1591-1601.

Burton, E., Lewin, G., Clemson, L., \& Boldy, D. (2014a). Determining the feasibility of a lifestyle activity program for inclusion in a restorative home care service: a pilot study. Activities, Adaption \& Aging, 38, 79-93.

Burton, E., Lewin, G., Clemson, L., \& Boldy, D. (2014b). Long-term benefits of a lifestyle exercise program for older people receiving a restorative home care service: a pragmatic randomized controlled trial. Healthy Aging \& Clinical Care Elderly, 6, 1-9.

Burton, E., Farrier, K., Lewin, G., Pettigrew, S., Hill, A. M., Airey, P., et al. (2017). Motivators and barriers for older people participating in resistance training: a systematic review. Journal of Aging and Physical Activity, 25(2), 311-324.

Clemson, L., \& Munro, J. (2015). Conceptual model of habit reforming to improve balance and prevent falls. Encyclopedia of geropsychology. Singapore: Springer. 
Clemson, L., Singh, M. F., Bundy, A., Cumming, R. G., Weissel, E., Munro, J., et al. (2010). LiFE pilot study: a randomised trial of balance and strength training embedded in daily life activity to reduce falls in older adults. Australian Occupational Therapy Journal, 57(1), 42-50.

Clemson, L., Fiatarone Singh, M.A., Bundy, A., Cumming, R.G., Manollaras, K., O'Loughlin, P., et al. (2012). Integration of balance and strength training into daily life activity to reduce rate of falls in older people (the LiFE study): randomised parallel trial. $B M J, 345$, e4547.

Clemson, L., Munro, J., \& Fiatarone Singh, M.A. (2014a). Lifestyle-integrated functional exercise (LiFE) program to prevent falls: trainer's manual. Sydney: University Press.

Clemson, L., Munro, J., \& Fiatarone Singh, M.A. (2014b). Lifestyle-integrated functional exercise (LiFE) program to prevent falls. Participant's manual. Sydney: University Press.

Clemson, L., Munro, J., Singh, M. F., Schwenk, M., \& Nerz, C. (2019). Trainer-Manual-Aktiv und Sicher Durchs Leben Mit Dem LiFE Programm. Berlin Heidelberg: Springer.

Costello, E., Kafchinski, M., Vrazel, J., \& Sullivan, P. (2011). Motivators, barriers, and beliefs regarding physical activity in an older adult population. Journal of Geriatric Physical Therapy, 34(3), 38-47.

Dillon, L., Clemson, L., Coxon, K., \& Keay, L. (2018). Understanding the implementation and efficacy of a home-based strength and balance fall prevention intervention in people aged 50 years or over with vision impairment: a process evaluation protocol. BMC Health Services Research, 18(1), 512.

Fleig, L., McAllister, M. M., Chen, P., Iverson, J., Milne, K., McKay, H. A., et al. (2016). Health behaviour change theory meets falls prevention: feasibility of a habit-based balance and strength exercise intervention for older adults. Psychology of Sport and Exercise, 22, 114-122.

Gibbs, J.C., McArthur, C., Milligan, J., Clemson, L., Lee, L., Boscart, V.M., et al. (2015). Measuring the implementation of a groupbased Lifestyle-integrated Function Exercise (Mi-LiFE) intervention delivered in primary care for older adults aged 75 years or older: a pilot feasibility study protocol. Pilot Feasibility Studies, 1,20

Gibbs, J.C., McArthur, C., Milligan, J., Clemson, L., Lee, L., Boscart, V.M., et al. (2019). Measuring the implementation of lifestyleintegrated functional exercise in primary care for older adults: results of a feasibility study. Canadian Journal on Aging/La Revue canadienne du vieillissement, 38(3), 350-366.

Granbom, M., Clemson, L., Roberts, L., Hladek, M.D., Okoye, S. M., Liu, M., et al. (2019). Preventing falls among older fallers: study protocol for a twophase pilot study of the multicomponent LIVE LiFE program. Trials, 20(1), 1-9.

Hu, Y. L., Keglovits, M., Somerville, E., Snyder, M., \& Stark, S. (2020). Translating a functional exercise program for fall prevention among diverse older adults in urban medically underserved areas: a mixed methods study. Journal of aging and physical activity, 1, 1-8.

Ivers, R. Q., Cumming, R. G., Mitchell, P., et al. (2003). Visual risk factors for hip fracture in older people. Journal of American Geriatric Society, 51, 356-363.

Jansen, C.-P., Nerz, C., Kramer, F., Labudek, S., Klenk, J., Dams, J., et al. (2018). Comparison of a group- delivered and individually delivered lifestyleintegrated functional exercise (LiFE) program in older persons: a randomized noninferiority trial. BMC Geriatrics, 1(1), 267.

Jansen, C.-P., Nerz, C., Labudek, S., Gottschalk, S., Kramer-Gmeiner, F., Klenk, J., Dams, J., König, H.-H., Clemson, L., Becker, C., \& Schwenk, M. (2021). Lifestyle-integrated functional exercise to prevent falls and promote physical activity: results from the LiFE-is-LiFE randomized noninferiority trial. Journal of Behavioral Nutrition and Physical Activity, 18(1), 1-12.

Keay, L., Saich, F., Clemson, L., Middlemiss, L., Johnson, J., Tumanik, H., et al. (2015). Feasibility and acceptability of orientation and mobility instructors delivering the LiFE falls prevention program to older people with vision impairment. International Journal of Orientation and Mobility $7(1), 22$.

Keay, L., Dillon, L., Clemson, L., Tiedemann, A., Sherrington, C., McCluskey, P., et al. (2018). PrevenTing falls in a high-risk, vision-impaired population through specialist ORientation and mobility services: protocol for the PlaTFORM randomised trial. Injury Prevention, 24(6), 459-466.

Kramer, F., Labudek, S., Jansen, C.-P., Nerz, C., Fleig, L., Clemson, L., et al. (2020). Development of a conceptual framework for a group-based format of the Lifestyle-integrated Functional Exercise (gLiFE) programme and its initial feasibility testing. Pilot and feasibility studies, 6 6.

Lally, P., \& Gardner, B. (2013). Promoting habit formation. Health Psychology Review, 7(1) 137-158.

Li, K., Comer, K., Huang, T., Schmidt, K., \& Tong, M. (2018). Effectiveness of a modified lifestyleintegrated functional exercise program in residential retirement communities-a pilot study. SAGE Open Nursing, 4, 1-12.

Merom, D., Pye, V., Macniven, R., van der Ploeg, H., Milat, A., Sherrington, C., et al. (2012). Prevalence and correlates of participation in fal prevention exercise/physical activity by older adults. Preventive Medicine, 55(6), 613-617.

Michie, S., Ashford, S., Sniehotta, F. F., Dombrowski, S. U., Bishop, A., \& French, D. P. (2011). A refined taxonomy of behaviour change techniques to help people change their physical activity and healthy eating behaviours: the CALORE taxonomy. Psychology \& Health, 26(11) 1479-1498.

Ounjaichon, S. (2020). Developing the Thai lifestyleintegrated functional exercise (TLiFE) programme: an intervention to prevent falls among older adults in Thailand. Manchester: The University of Manchester

Reicherzer, L., Kramer-Gmeiner, F., Labudek, S., Jansen, C.-P., Nerz, C., Nystrand, M. J., et al. (2021). Group or individual lifestyle-integrated functional exercise (LiFE)? A qualitative analysis of acceptability. BMC Geriatrics, 21(1), 93.

Ryan, R., \& Deci, E. (2000). Self-determination theory and the facilitation of intrinsic motivation, social development, and well-being. American Psychologist, 55(1), 8-78.

Schwarzer, R. (2008). Modeling health behavior change: How to predict and modify the adoption and maintenance of health behaviors. Applied Psychology, 57(1), 1-29.

Schwenk, M., Bergquist, R., Boulton, E., Van Ancum, J.M., Nerz, C., Weber, M., et al. (2019). The adapted lifestyle-integrated functional exercise program for preventing functional decline in young seniors: development and initial evaluation. Gerontology, 65(4), 362-374.

Sherrington, C., Fairhall, N., Wallbank, G., Tiedemann, A., Michalef, Z. A., Howard, K., et al. (2020). Exercise for preventing falls in older people living in the community: an abridged, Cochrane systematic review. British Journal of Sports Medicine, 54(15), 885-891.

Szanton, S. L., Clemson, L., Liu, M., Gitlin, L. N., Hladek, M. D., LaFave, S. E., et al. (2021). Pilot outcomes of a multicomponent fall risk program integrated into daily lives of community-dwelling older adults. Journal of Applied Gerontology, 40(3), 320-327.

Taraldsen, K., Mikolaizak, A.S., Maier, A. B., Boulton, E., Aminian, K., Van Ancum, J., et al. (2019). Protocol for the PreventIT feasibility randomised controlled trial of a lifestyle-integrated exercise intervention in young older adults. BMJ open, 9(3), e23526.

Taraldsen, K., Mikolaizak, A. S., Maier, A. B., Mellone, S., Boulton, E., Aminian, K., et al. (2020). Digital technology to deliver a lifestyle-integrated exercise intervention in young seniors-the preventIT feasibility randomized controlled trial. Frontiers in Digital Health, 2, 10.

Van Alphen, H. J., Volkers, K.M., Blankevoort, C.G., Scherder, E. J., Hortobagyi, T., \& van Heuvelen, M.J. (2016). Older adults with dementia are sedentary for most of the day. PLoS One, 11(3), e152457.

Weber, M., Belala, N., Clemson, L., Boulton, E., HawleyHague, H., Becker, C., et al. (2018). Feasibility and effectiveness of intervention programmes integrating functional exercise into daily life of older adults: a systematic review. Gerontology, 64(2), 172-187.

World Health Organization (2021). Falls fact sheet WHO. https://www.who.int/news-room/factsheets/detail/falls. Last access: 21.08.2021

Zhang, W., Schwenk, M., Mellone, S., Paraschiv-lonescu, A., Vereijken, B., Pijnappels, M., et al. (2018). Complexity of daily physical activity is more sensitive than conventional metrics to assess functional change in younger older adults. Sensors, 18(7), 2032 